\title{
Anomalous origin of the right coronary artery from the pulmonary artery (ARCAPA) in an infant with a heart murmur. Case report
}

\author{
Melina J. Saavedra, M.D. ${ }^{a}$, Javier Mozzi, M.D. ${ }^{a}$, Natalia Napoli, M.D. ${ }^{a}$, Alejandra Villa, M.D. ${ }^{a}$, \\ Jorge Barretta, M.D. ${ }^{b}$ and Pablo Marantz, M.D. ${ }^{a}$
}

\begin{abstract}
The anomalous origin of the right coronary artery from the pulmonary artery (ARCAPA) is a rare entity that has an incidence of $0.002 \%$ and can potentially cause sudden death. Unlike to the anomalous origin of the left coronary artery from the pulmonary artery (ALCAPA), usually presented with myocardial ischemia and heart failure; the diagnosis of ARCAPA is often incidental during evaluation of a heart murmur.

We report a case of a 6 months old female patient referred to us with a cardiac murmur and a suspicion of coronary fistula. For a proper diagnostic assessment an echocardiogram, a computed tomography angiography and a cardiac catheterization were requested. A small ostium secundumtype atrial septal defect (ASD) and an ARCAPA were revealed. At 11 months old, the patient was scheduled for corrective cardiovascular surgery.

The aim of this report is to describe a low incidence and potentially fatal disease.

Key words: $A R C A P A$, coronary anomaly, ALCAPA, myocardial ischemia, sudden death.
\end{abstract}

http:/ / dx.doi.org/10.5546/ aap.2018.eng.e789

To cite: Saavedra MJ, Mozzi J, Napoli N, Villa A et al. Anomalous origin of the right coronary artery from the pulmonary artery (ARCAPA) in an infant with a heart murmur. Case report. Arch Argent Pediatr 2018;116(6):e789-e792. a. Department of Pediatrics. Division of Pediatric Cardiology.

b. Division of Pediatric Cardiovascular Surgery. Hospital Italiano de Buenos Aires.

E-mail address:

Melina J. Saavedra, M.D.: melina.saavedra@hiba.org.ar

Funding: None.

Conflict of interest: None.

Received: 5-17-2018

Accepted: 7-31-2018

\section{INTRODUCTION}

Congenital coronary artery anomalies are rare entities in the general population. ${ }^{1}$ ARCAPA is an extremely rare disease with an incidence of $0.002 \% .^{2}$ Associated congenital heart defects have been previously reported in one third of the patients being aortopulmonary window and tetralogy of Fallot the most commonly manifestations. ${ }^{2-5}$

Previous reports in the literature describe approximately 100 cases of ARCAPA of which $50 \%$ percent were incidental findings during a heart murmur evaluation. In addition some patients present with signs of ischemia in the electrocardiogram (ECG) evaluation. ${ }^{2-4,6,7}$

Abnormal aortic origin of coronary arteries is a life-threatening congenital disease that can lead to sudden death, thereby surgical correction would be recommended still for all diagnosed cases, even in asymptomatic patients. ${ }^{4,-9}$

\section{CASE REPORT}

We report a case of a 6-month-old female child referred with a clinical suspicion of coronary fistula. She was asymptomatic and was assessed by a heart murmur in her hometown clinic.

Based on World Health Organization's standards, the patient was at the $97^{\text {th }}$ percentile for weight and $6^{\text {th }}$ percentile for height. Past medical history was unremarkable, except for an episode of bronchiolitis at 4 months age which required hospital admission and oxygen therapy. A systolic heart murmur grade $2 / 6$ best heard at lower left sternal border was detected during cardiovascular examination. The echocardiogram showed an ostium secundum atrial septal defect (ASD) in addition to a presumptive image of coronary fistula.

She was referred to our Pediatric Cardiology Division for a complete diagnostic assessment. Transthoracic echocardiogram was performed, revealing the dilatation of both coronary arteries. In addition, a suggestive image of multiple fistulas with connection to the right ventricle was observed, together with a small ASD without dilatation of right atrium and ventricle. 
Cardiac catheterization was subsequently performed and the right coronary artery was not observed in the aortic root during contrast media injection (Figure 1). Pulmonary artery diastolic pressure was observed to be slightly increased $(25 / 16 \mathrm{mmHg}$, mean $19 \mathrm{mmHg})$ and the left ventricular end diastolic pressure was $12 \mathrm{mmHg}$. Moreover, the left coronary artery was dilated

FIGURE 1. Angiography

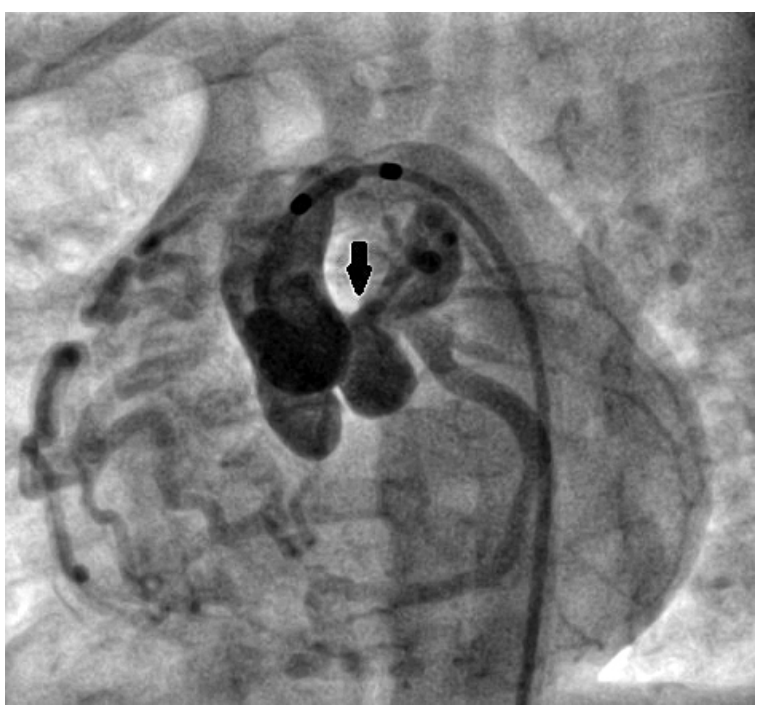

Dilated left coronary artery (black arrow). The right coronary ostium was not observed in the aortic root during contrast media injection. Collateral circulation development. with connection to the right coronary territory and subsequent drainage in the main pulmonary artery. Mild pulmonary overcirculation was revealed with Qp/Qs 1.2:1. During contrast media injection into the pulmonary artery, the right coronary artery origin could not be appreciated with certainty.

Given the complexity of the coronaries anatomy in this patient, a multislice computed tomography angiography was performed to achieve the diagnosis. The right coronary artery anomalous origin was detected to emerge from the main pulmonary artery, $8.6 \mathrm{~mm}$ above the valve leaflets in anterior and right position. It passed by front of the aorta towards the right atrioventricular groove. The left coronary artery was dominant and originated in the left coronary aortic sinus. Abundant collateral circulation was revealed between the left anterior descending and circumflex arteries and the right coronary artery. ARCAPA diagnosis was thereby confirmed (Figure 2).

A grand round conference between pediatrics cardiologists and pediatric cardiac surgeons was scheduled and therapeutic strategies were discussed. Surgical correction was indicated as the best treatment plan.

Baseline ECG was within normal limits

Surgical reimplantation of the right coronary artery into the aorta and ASD closure were performed at 11 months of age. An intraoperative transesophageal echocardiogram revealed a

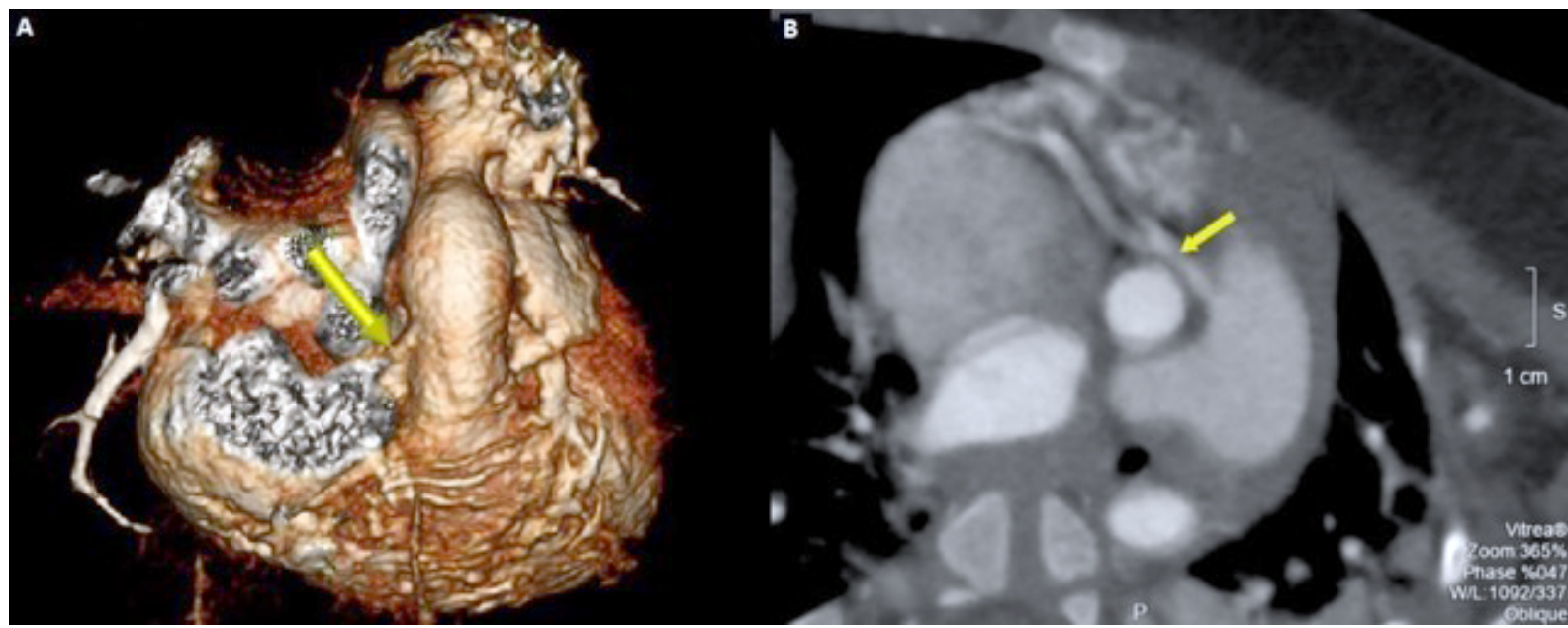

A. Three dimensional reconstruction that shows right coronary artery emerging from the main pulmonary artery.

B. Short axis view. Right coronary artery emerging from the main pulmonary artery. 
dilated left coronary artery arising from the aorta and right coronary artery emerging from the main pulmonary artery in a lateral and right-sided position; the presence of abundant collateral circulation was observed (Figure 3). The surgery lasted 207 minutes with extracorporeal circulation requirement during 66 minutes and aortic clamping for 35 minutes. Inotropic support consisted of milrinone $0.5 \mathrm{mcg} / \mathrm{kg} / \mathrm{min}$ and adrenaline $0.05 \mathrm{mcg} / \mathrm{kg} / \mathrm{min}$. Mechanical ventilation and adrenaline infusion were weaned off 5 hours after surgery. Milrinone infusion was provided for 48 hours and then she was started enalapril PO.

The patient did not develop any post-operative complications. A follow-up echocardiogram revealed the right coronary artery implanted in the aortic artery with a laminar flow and preserved biventricular function. The patient was discharged on the third postoperative day, and was prescribed with enalapril $0.1 \mathrm{mg} / \mathrm{kg}$ and furosemide $2 \mathrm{mg} /$ $\mathrm{kg}$. She currently continues asymptomatic in follow-up visits with the Pediatric Cardiology Division.

\section{DISCUSSION}

Congenital coronary arteries anomalies are vascular anatomical defects which are present from birth. ARCAPA is a rare medical condition, first described in 1885 by Brooks. ${ }^{10}$ ARCAPA can be present in structurally normal hearts or it can be associated with other congenital heart defects in $30 \%$ of the cases being aortopulmonary window and tetralogy of Fallot the most reported entities. $^{2-5}$ Our patient exhibited ARCAPA associated with ASD, which has been reported less frequently in the literature.

About 100 cases of ARCAPA including pediatric and adult patients have been previously described in the literature with varied clinical symptoms. ${ }^{5}$ Most of these patients were incidentally diagnosed during a heart murmur evaluation. ${ }^{2,4,6,7}$ Unlike ALCAPA, known for early clinical manifestations such as mitral regurgitation and signs of anterolateral myocardial infarction, the majority of patients with ARCAPA tend to be asymptomatic. However, some patients have been described to show signs of myocardial ischemia on the ECG at the time of diagnosis. ${ }^{3}$

In this specific case, the diagnosis was made in an asymptomatic patient, who was evaluated for the presence of a heart murmur without signs of myocardial ischemia on the ECG.

Less frequently, ARCAPA may develop symptoms due to the phenomenon of coronary steal that occurs from the left to the right coronaries through the development of collateral circulation. In such cases, patients may present with symptoms of myocardial ischemia such as chest pain, dyspnea, fatigue, congestive heart failure, myocardial infarction, and even sudden death. ${ }^{4-6,8}$ The severity of symptoms depends on several aspects, like the type of anomaly, the impact on the oxygen delivery to the myocardium, the direction of blood flow and the development of collateral circulation. . $^{2,4,6,7}$

Some other cardiac defects associated with ARCAPA can be protective, such as stenosis at the origin of an anomalous right coronary artery, left dominant coronary circulation and left to right shunts (ventricular septal defects, patent ductus arteriosus, or aortopulmonary window)., ${ }^{2,11}$ Increased pressure in the pulmonary artery is associated with a lower steal from the coronary circulation to the pulmonary artery and also may also provide a greater anterograde flow from

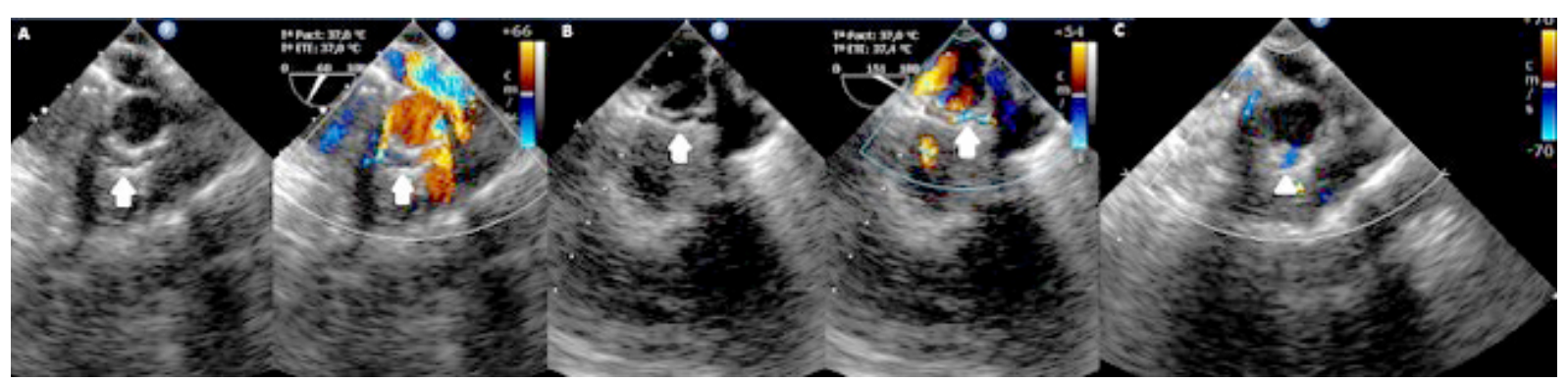

A y B. Shows right coronary artery emerging from the main pulmonary artery (white arrow).

C. Post-operative image that revealed re-implanted right coronary artery into aortic root. (white arrowhead). 
the pulmonary artery into the right coronary artery. ${ }^{11}$ In our patient, the left dominant coronary circulation could have exerted a protective effect.

The methods of diagnosis of ARCAPA depended on period of the cases reported. Before 1985, the diagnosis was mostly made by autopsy, angiography or during cardiovascular surgery. ${ }^{2,4}$ Currently, non- invasive diagnostic techniques such as transthoracic echocardiogram can be used to detect ARCAPA. Dilatation of the left coronary artery, collateral circulation development with the appearance of fistulas can be observed. Moreover, the visualization of an anomalous origin of the right coronary artery with retrograde flow to the main pulmonary artery are characteristic findings. ${ }^{5,6}$ However, two dimensional images can have limitations to confirm ARCAPA diagnosis. ${ }^{5}$ Multislice computed tomography angiography and cardiac magnetic resonance are more accurate non-invasive diagnostic methods that allow three dimensional reconstruction of the region of interest. These methods are becoming increasingly popular imaging modalities that allow physicians to achieve a precise diagnosis. ${ }^{5}$

In patients diagnosed with ARCAPA, given the coronary steal phenomenon and high risk of myocardial ischemia, surgical correction with reimplantation of right coronary artery into aortic root is strongly recommended, even in asymptomatic patients. ${ }^{2,4,7,8}$ Coronary reimplantation reestablishes dual coronary ostium circulation decreasing the risk for sudden death. ${ }^{8}$ At 11 months of age, corrective cardiovascular surgery was performed without complications.

\section{CONCLUSIONS}

ARCAPA is a rare, life-threatening coronary anomaly. Its incidence probably remains underestimated given the proportion of asymptomatic patients that don't seek medical attention. Due to the increased incidence in patients with co-existing congenital heart defects, it is very important to properly assess the coronary arteries anatomy to detect any potential anomaly.
Current non-invasive imaging techniques such as computed tomography angiography or cardiac magnetic resonance are available to perform an accurate diagnosis.

Surgical reimplantation of the right coronary artery into the aorta to re-establish dual coronary circulation is recommended. This is strongly encouraged even in asymptomatic patients, in order to reduce the risk for sudden death.

\section{REFERENCES}

1. Angelini P, Velasco JA, Flamm S. Coronary anomalies: incidence, pathophysiology, and clinical relevance. Circulation. 2002;105(20):2449-54.

2. Williams IA, Gersony WM, Hellenbrand WE. Anomalous right coronary artery arising from the pulmonary artery: A report of 7 cases and a review of the literature. Am Heart J. 2006;152(5):1004.e9-17.

3. RadkePW, Messmer BJ, Haager PK, Klues HG. Anomalous origin of the right coronary artery: preoperative and postoperative hemodynamics. Ann Thorac Surg. 1998;66(4):1444-9.

4. Kim K, Jo E, Yu J, Kil H. Anomalous right coronary artery from pulmonary artery discovered incidentally in an asymptomatic young infant. Korean J Pediatr. 2016;59(Suppl 1):S80-3.

5. Gilmour J, Kafka H, Ropchan G, Johri AM. Anomalous right coronary artery: a multimodality hunt for the origin. Case Rep Cardiol. 2011;2011:286598.

6. Afolabi-Brown O, Witzke C, Moldovan R, Pressman G. A Different Kind of Christmas Tree: Anomalous Origin of the Right Coronary Artery from the Pulmonary Artery (ARCAPA). Echocardiography. 2014;31(2):e52-4.

7. García Manzano PR, Domínguez P, Merino L, et al. Origen anómalo de arteria coronaria derecha desde arteria pulmonar: reimplante directo en aorta en un niño de 8 meses de edad. Rev Fed Arg Cardiol. 2010;39(2):133-5.

8. Bregman D, Brennan JF, Singer A, et al. Anomalous origin of the right coronary artery from the pulmonary artery. J Thorac Cardiovasc Surg. 1976;72(4):626-30.

9. Veselka J, Widimský P, Kautzner J. Reimplantation of anomalous right coronary artery arising from the pulmonary trunk leading to normal coronary flow reserve late after surgery. Ann Thorac Surg. 2003;76(4):1287-9.

10. Brooks HS. Two cases of an abnormal coronary artery of the heart arising from the pulmonary artery: with some remarks upon the effect of this anomaly in producing cirsoid dilatation of the vessels. J Anat Physiol. 1885;20(Pt 1):26-9.

11. Ramani J, Ananthanarayanan C, Pujara J, et al. Intramural Anomalous Right Coronary Artery From the Main Pulmonary Artery. World J Pediatr Congenit Heart Surg. 2017;8(6):745-9. 